\title{
Dangers of using the edges of the Brillouin zone
}

\author{
R. V. Craster, ${ }^{1}$ T. Antonakakis, ${ }^{1,2}$ M. Makwana, ${ }^{1}$ and S. Guenneau ${ }^{3}$ \\ ${ }^{1}$ Department of Mathematics, Imperial College London, London SW7 2AZ, United Kingdom \\ ${ }^{2}$ European Organization for Nuclear Research, CERN CH-1211, Geneva 23, Switzerland \\ ${ }^{3}$ Institut Fresnel, UMR CNRS 7249, University of Aix Marseille, Marseille, France
}

(Received 14 May 2012; published 21 September 2012)

\begin{abstract}
In solid-state physics, including photonics and wherever periodic lattice structures occur, it is essential to establish the fundamental features associated with wave propagation through the lattice: This is achieved using Bloch waves, the reciprocal lattice, and the reduction, using periodicity, to consider the irreducible Brillouin zone. A general approach, although widely accepted as not being perfectly legitimate, is to plot the dispersion relations around the edges of the Brillouin zone. We show definitively that this can be dangerous and that an important mode of practical significance is missed if this is done in too cavalier a fashion: This missing mode is illustrated for the design of endoscopes based on spring-mass (discrete) periodic structures and photonic crystals.
\end{abstract}

DOI: 10.1103/PhysRevB.86.115130

PACS number(s): 42.70.Qs, 42.25.Fx, 43.40.+s, 62.30.+d

\section{INTRODUCTION}

The generally accepted folklore is that plotting the dispersion relations around the edges of the Brillouin zone $e^{1,2}$ is broadly sufficient to identify the extent of stop bands, as the maxima and minima almost always occur there. ${ }^{3}$ Some mathematical literature ${ }^{4,5}$ explicitly constructs counterexamples; nonetheless it is almost always the case that the edges of the Brillouin zone contain the essential information sufficient for most purposes. However, as we describe, one can actually overlook a mode, or modes, that arises from a path within the Brillouin zone and that exciting this mode, as shown in Fig. 1, is of interest; it is almost certainly present in many periodic structures already analyzed and has been missed. For clarity we treat the simplest possible cases: a square array of circular infinite conducting cylindrical holes in transverse electric (TE) polarization (i.e., a longitudinal magnetic field orthogonal to the plane of periodicity) [see Fig. 2(b)] and a square array of simple masses and springs [see Fig. 2(a)]. However, our analysis can be applied with some ad hoc changes to other areas of physics whenever some periodicity occurs, such as solid-state physics ${ }^{2,6}$ or the theory of composites. ${ }^{7,8}$

\section{FORMULATION OF CONTINUOUS MODEL}

We consider the Helmholtz equation as a generic model for wave propagation which could, with appropriate notational and linguistic changes, hold for acoustic, electromagnetic, water, or out-of-plane elastic waves and encompass many possible physical applications. We solve

$$
\frac{\partial^{2} u}{\partial x_{1}^{2}}+\frac{\partial^{2} u}{\partial x_{2}^{2}}+\Omega^{2} u=0,
$$

for $u\left(x_{1}, x_{2}\right)$ on the square cell $-1<x_{1}, x_{2}<1$.

In the context of optics, the unknown $u$ in Eq. (1) is the longitudinal component of the electric field $H_{z}$ in TE polarization and the spectral parameter $\Omega^{2}$ is associated with $\omega^{2} \varepsilon\left(x_{1}, x_{2}\right) / c^{2}$ wherein $\omega$ is the electromagnetic wave frequency, $\varepsilon$ is the relative permittivity, and $c$ is the speed of light in a vacuum.

For waves through an infinite, perfect, doubly periodic medium based upon a square lattice, one invokes Bloch's theorem ${ }^{1,2,9,10}$ and then simply considers the square cell with quasiperiodic Bloch conditions applied to the edges:

$$
\begin{gathered}
u\left(1, x_{2}\right)=e^{\mathrm{i} \kappa_{1}} u\left(-1, x_{2}\right), \quad u_{x_{1}}\left(1, x_{2}\right)=e^{\mathrm{i} \kappa_{1}} u_{x_{1}}\left(-1, x_{2}\right), \\
u\left(x_{1}, 1\right)=e^{\mathrm{i} \kappa_{2}} u\left(x_{1},-1\right), \quad u_{x_{2}}\left(x_{1}, 1\right)=e^{\mathrm{i} \kappa_{2}} u_{x_{2}}\left(x_{1},-1\right),
\end{gathered}
$$

and introduce the Bloch wave vector $\kappa=\left(\kappa_{1}, \kappa_{2}\right)$ characterizing the phase shift as one moves from one cell to the next. This Bloch problem is solved numerically, and dispersion relations that link the frequency and Bloch wave number are deduced. As is often presumed in solid-state physics, ${ }^{1}$ only a limited range of wave numbers are considered, namely the wave numbers along the right-angled triangle $\Gamma X M$ shown in the irreducible Brillouin zone in Fig. 2(c). There are, however, some exceptions to this rule, such as operators on graphs ${ }^{5}$ and Bloch waves in periodic acoustic ${ }^{4}$ and elastic ${ }^{11}$ strips.

The computed dispersion curves for discrete (Fig. 3) and continuous (Fig. 4) structures illustrate several interesting features: stop bands for which wave propagation is not possible and striking regions of flat dispersion curves for which the group velocity is zero and features of slow sound or light occur. ${ }^{12}$ The missing mode illustrated in Fig. 1 is excited at a frequency close to this flat dispersion curve; it is perfectly flat in the discrete mass-spring system (cf. dashed curve in Fig. 3) and almost so in the continuum analog of holes in a square array (cf. dashed curve in Fig. 4). The hole size is realistic and could be manufactured; moving to artificially large hole sizes pushes the analogy of holes and masses toward being explicit (Sec. V). Excitation at, or very close, to the frequency predicted leads to a diagonal cross of oscillations that resemble a standing wave in Fig. 1. Crucially this standing wave has directionality that can be identified from the wave-number description in the Brillouin zone shown in Fig. 2.

The essence of our argument is that in, say, a square lattice one can choose different elementary cells. The obvious and natural elemental cell is a single hole, or mass, in a square cell [cf. Fig. 2(a)] with Bloch conditions applied at the edges of the cell. Of course, one could choose other cells, such as one with four holes, or masses; naively one would expect to obtain the same dispersion relations, modulo some folding due to the periodicity, in both cases. The Brillouin zones for 
(a) $\left|y_{n m}\right|$

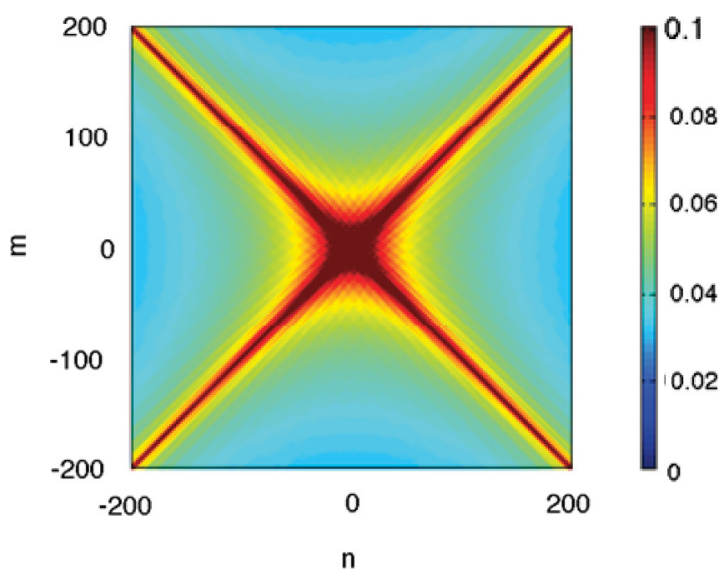

(b) lul

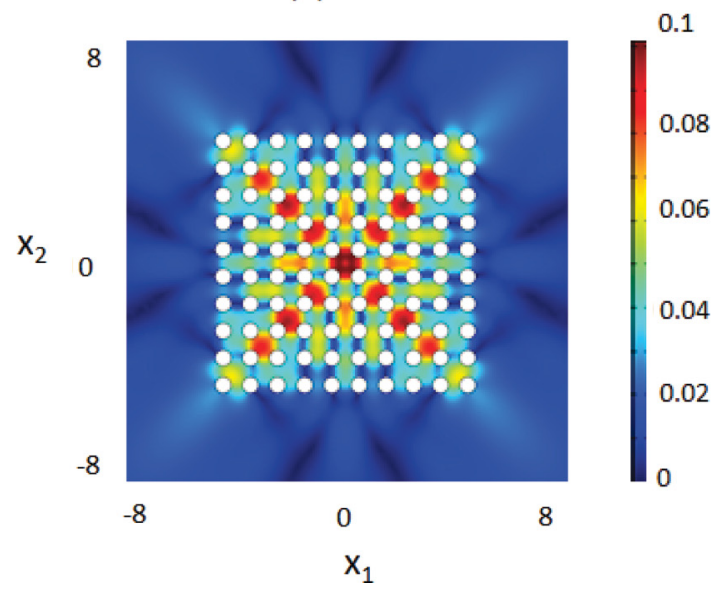

FIG. 1. (Color online) (a) A diagonal cross for a discrete massspring square periodic array; the standing wave is excited by a forcing of normalized frequency $\Omega=1.99$ at the origin for $1000 \times 1000$ masses surrounded by a layer of discrete perfectly matched layers (PMLs) 100 masses deep. (b) Same but for an electric line source of frequency $\Omega=2.24$ located at $(0,0)$ radiating in an isotropic dielectric medium with a square array (pitch 1$)$ of $10 \times 10$ infinite conducting cylinders (radius $r=0.3$ ) in TE polarization (i.e., Neumann boundary conditions), surrounded by continuous PMLs outside the square region $-8<x_{j}<8, j=1,2$.

each cell are shown in Fig. 2(c) and, as one would expect, the zone corresponding to the single mass cell is fundamental as it subsumes that of the four-cell system. However, if one plots the dispersion curves going around the exterior of the larger (single-mass) zone, one never plots the dispersion relations along $M X^{\prime}$ in the interior of the larger cell: This line, even after the reflections of periodicity, always lies in the interior of the larger one. This would be of little consequence if the modes it illustrated were disinteresting; however they are of interest and are the diagonal cross modes. Observing the dispersion curves in Figs. 3 and 4, one sees that the naive approach is broadly true, except that suddenly a "new" mode appears in the four-hole (mass) cases (shown as the dashed lines between $M$ and $X^{\prime}$ ). Importantly, this is perfectly flat in the massspring case and nearly so for the holes. This is even more

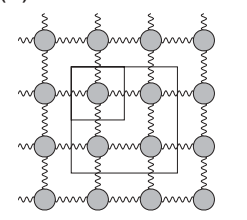

(a) Lattice of masses (b) Square array of cylinders

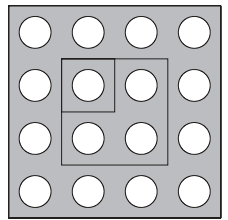

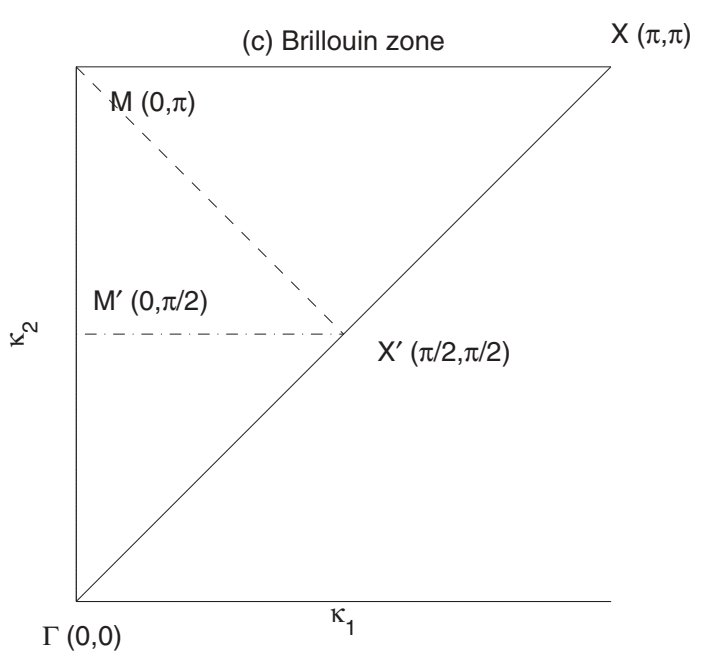

FIG. 2. The simple discrete mass-spring system shown in panel (a) with the single-mass cell and four-mass cell shown. An array of square cells with cylindrical inclusions is represented in panel (b). Panel (c) shows the Brillouin zones used for the single-mass or cylinder cell $\Gamma, X, M$ and for the four-mass or cylinder cell $\Gamma, X^{\prime}, M^{\prime}$.

clear when one observes the isofrequency contours for the acoustic branch as shown in Fig. 5: The perfect flat band for the mass-spring system corresponds to the straight diagonal line in Fig. 5(a), and although the corresponding path for the circular holes is no longer perfectly straight, the physical phenomena from the mass-spring system persist. The flat curve has been spotted, in a different context, for the mass-spring system ${ }^{13,14}$ and in that system its presence can be derived analytically; as a result the diagonal cross has been seen numerically in simple systems. ${ }^{13,15}$ Such flat modes, or nearly flat modes, are of considerable interest in the context of slow light and slow sound. ${ }^{12}$

\section{FORMULATION OF DISCRETE MODEL}

The discrete mass system, assuming identical unit masses and spring constants, with the single-mass cell boils down to the difference equation

$$
y_{n+1, m}+y_{n-1, m}+y_{n, m+1}+y_{n, m-1}-4 y_{n, m}+\Omega^{2} y_{n, m}=0
$$

as the discrete Helmholtz equation [counterpart of Eq. (1)], $\Omega$ being the frequency.

The Floquet-Bloch conditions ${ }^{16}$

$$
y_{n+N, m+M}=\exp \left(i\left[N \kappa_{1}+M \kappa_{2}\right]\right) y_{n, m}
$$

are a discrete counterpart of Eqs. (2) and (3) (here $N, M$ are integers); the resulting dispersion relation is immediate,

$$
\Omega^{2}=4-2\left(\cos \kappa_{1}+\cos \kappa_{2}\right),
$$


(a) Single mass cell

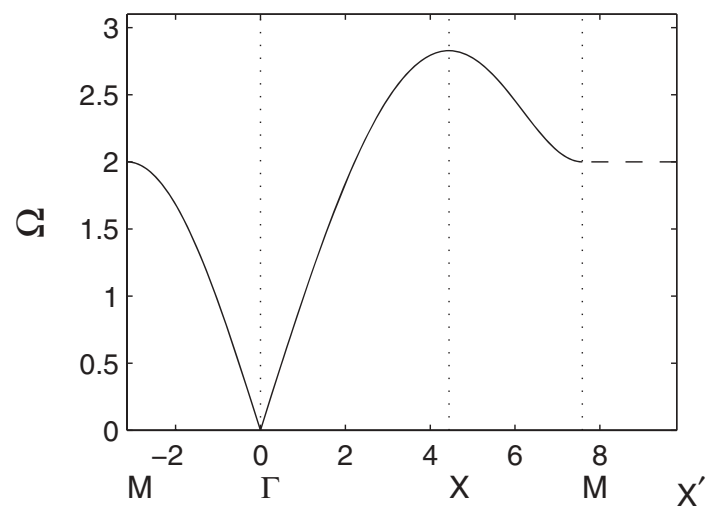

(b) 4 mass cell

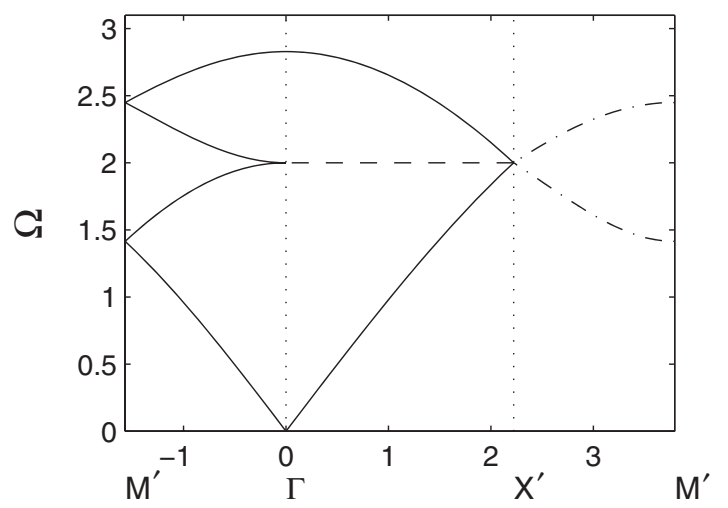

FIG. 3. Bloch dispersion curves around the edges of the Brillouin zone shown in Fig. 2 for the spring-mass model. Panel (a) shows the dispersion curves for the single-cell model and panel (b) shows them for the four-cell system: The latter has a flat mode between $\Gamma$ and $X^{\prime}$ that is absent from panel (a) if one does not consider the $M X^{\prime}$ path. This missing mode corresponds to panel (a) in Fig. 1.

with $0 \leqslant \kappa_{1}, \kappa_{2} \leqslant \pi$ and is shown in Fig. 4(a). The four-mass system results in four coupled difference equations which have solutions

$$
\begin{gathered}
2\left(\cos \kappa_{1}+\cos \kappa_{2}\right) \pm\left(4-\Omega^{2}\right)=0 \\
2\left(-\cos \kappa_{1}+\cos \kappa_{2}\right) \pm\left(4-\Omega^{2}\right)=0
\end{gathered}
$$

with $0 \leqslant \kappa_{1}, \kappa_{2} \leqslant \pi / 2$, and the four positive roots of these correspond to the four paths one would get from reflecting the smaller triangle $\Gamma X^{\prime} M^{\prime}$ to fill the larger one. In particular, the missing dashed line, which is a double root, comes from the path along $X^{\prime} M$ shown as dashed in Fig. 2(c). Also shown in Fig. 2(c) is another line $M^{\prime} X^{\prime}$ corresponding to the dot-dashed curves in Fig. 3; repeated roots also appear but are of less interest. One can tease out the asymptotic structure of the dispersion relations near the edges of the Brillouin zone and then to relate these to asymptotic partial differential equations ${ }^{14}$ that can be used to represent the solutions on a long scale. ${ }^{17}$

Importantly, this analysis of discrete models underpins the physics of the missing mode in continuous systems such as arrays of infinite conducting circular cylinders for TE waves in
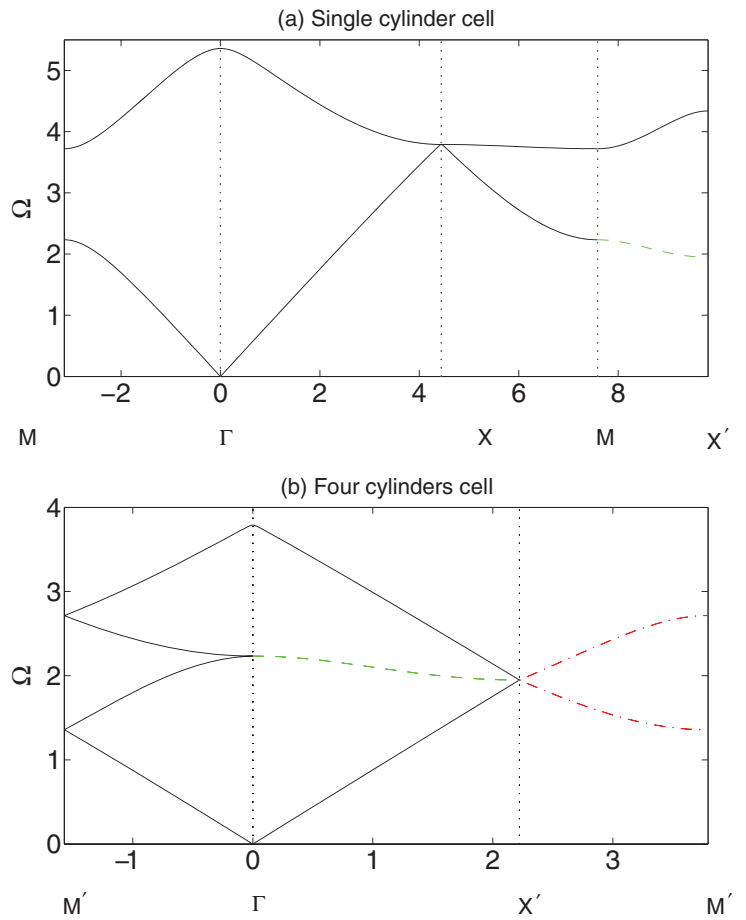

FIG. 4. (Color online) Bloch dispersion curves around the edges of the Brillouin zone shown in Fig. 2 for the continuous model. Panel (a) shows the dispersion curves for the single cell with an infinite conducting cylinder of radius $r=0.3$ and panel (b) for the four cell system: The latter has a nearly flat mode between $\Gamma$ and $X^{\prime}$ absent from panel (a) if one does not consider the $M X^{\prime}$ path.

optics or stress-free cylinders for anti-plane-shear ( $\mathrm{SH}$ ) waves in acoustics: Discrete structures are often used in mechanics as asymptotic models of densely packed composites, ${ }^{8}$ and one can then invoke the one-to-one correspondence between the Helmholtz equation governing these types of acoustical (e.g., SH) and optical (e.g., TE) waves. In Sec. V we include limiting cases showing nearly perfect agreement between the dispersion curves in Fig. 3 for the discrete lattice structure and dispersion curves for close-to-touching cylinders, whereby a completely flat mode is obtained along the $\Gamma X^{\prime}$ edge, as in Fig. 3. However, having in mind a practical design of an endoscope, we initially constrain ourselves to cylinders of a moderate radius, and therefore the dashed curve along the $\Gamma X^{\prime}$ edge still displays some dispersion in Fig. 4. The dispersion curves in Figs. 3 and 4 nonetheless share many common features, such as a quasi-identical dispersion for the dot-dashed curves along the $X^{\prime} M^{\prime}$ edge of the Brillouin zone for the periodic cell with four masses and four cylinders.

\section{PRACTICALLY IMPLEMENTABLE DESIGN}

We finally propose a practically implementable design of endoscope using the dispersionless feature of the missing mode along the $\Gamma X^{\prime}$ edge of the Brillouin zone for discrete (see Fig. 6) and continuous (see Fig. 7) periodic structures. In the former, the trajectory of the wave emitted by an excited 
(a)

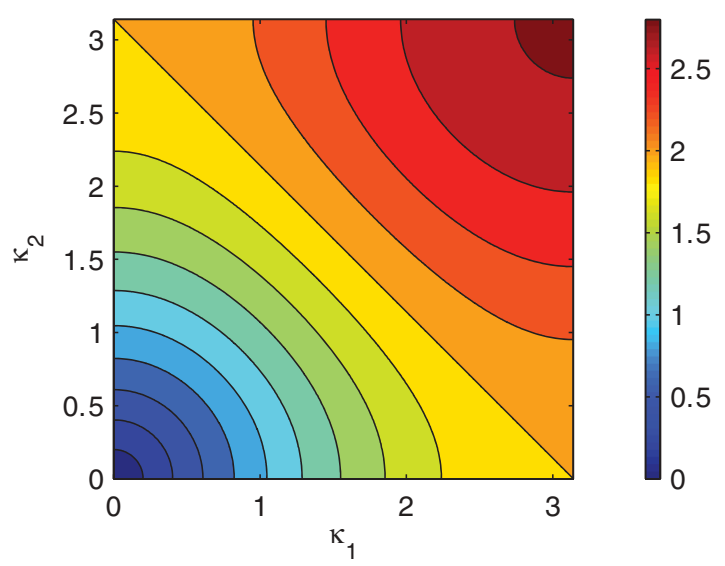

(b)

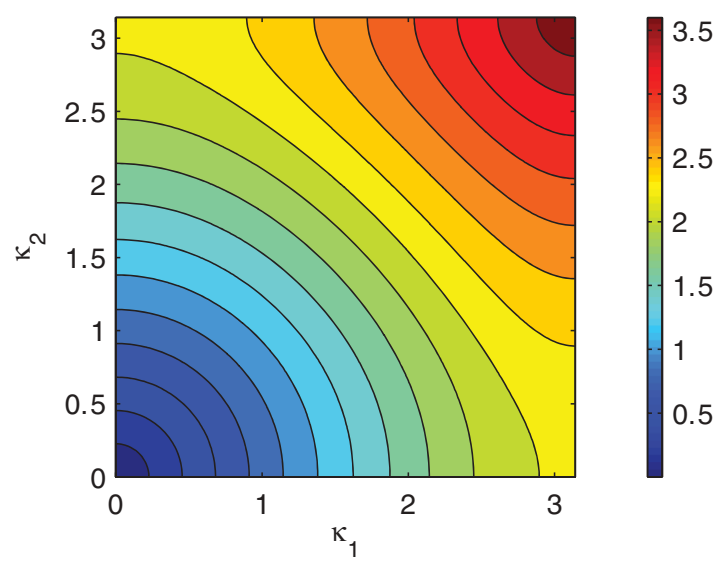

FIG. 5. (Color online) Isofrequency curves for the acoustic branch of (a) the spring-mass model and (b) of the cylindrical holes of radius 0.3 .

mass zigzags throughout the array of spring and masses, as it travels along $x_{1}= \pm x_{2}$ directions and reflects internally when the masses change; the wave trajectories resemble those of geometrical ray optics (valid in the limit of short wavelengths) although we are in a Bragg regime. A sharp focus point is created, reminiscent of negative refraction from a ray picture with alternating positively and negatively refracting slabs forming a one-dimensional array of Pendry-Veselago

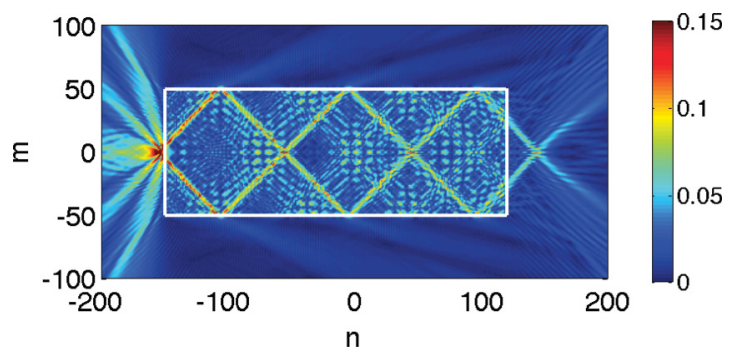

FIG. 6. (Color online) A square array of point masses, with mass 1.5 with a rectangular inset of unit masses from $n=-150$ to 120 and $m=-50$ to 50 . A mass at $(-155,0)$ is excited with an image clearly visible at $(+150,0)$; the normalized frequency of excitation is $\Omega=1.98$ very close to the flat band in Fig. 3 .

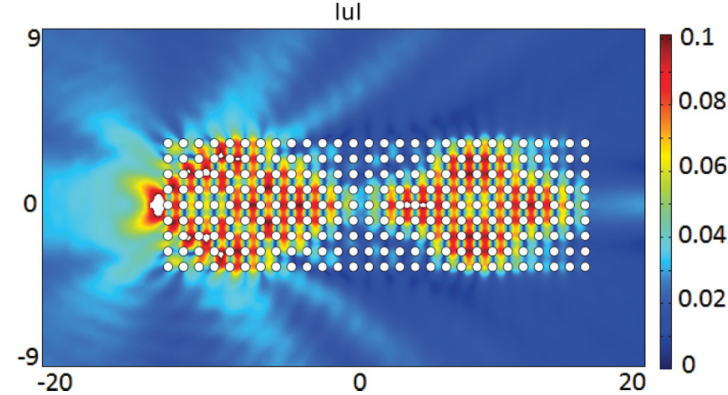

FIG. 7. (Color online) Endoscope effect with the missing mode in a square array (pitch 1) of $28 \times 9$ infinite conducting circular holes of radius $r=0.3$ for an electric line source of normalized frequency $\Omega=2.23$ located at point $(-12,0)$.

flat lenses. ${ }^{18}$ One can clearly see that the image of the source through the endoscope is deeply subwavelength. When we try to reproduce this discrete system paradigm with the array of cylinders, we only achieve this endoscope effect to certain extent: The image of an electric line source radiating at the frequency of the missing mode produces a photonic jet ${ }^{19}$ rather than a focusing point. We attribute this lack of precise focusing to the dispersive nature of the dashed curve in Fig. 4, which is the continuous model counterpart of the completely flat dashed curve in Fig. 3. One improves upon this situation by enlarging the cylinders (Sec. V) and/or by rotating the array (Sec. VI).

(a) Single cylinder cell

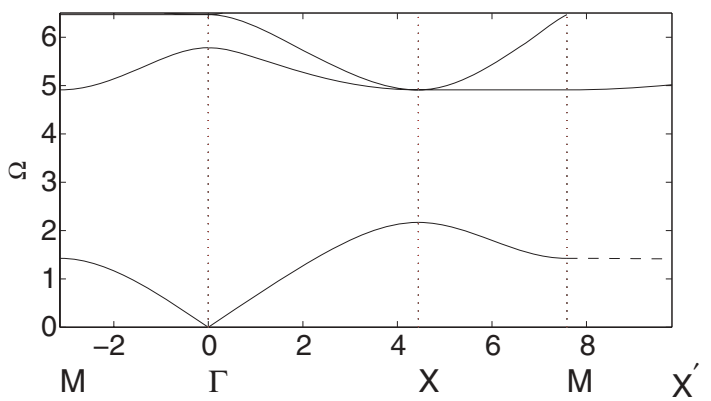

(b) Four cylinders cell

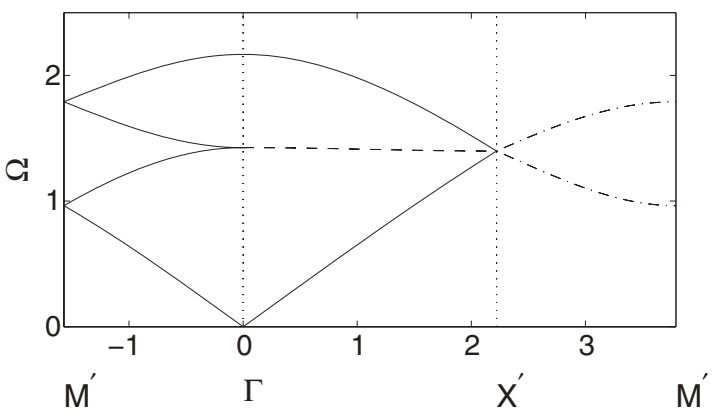

FIG. 8. (Color online) Bloch dispersion curves around the edges of the Brillouin zone shown in Fig. 2 for almost touching cylinders of radius 0.475 . Panel (a) shows the dispersion curves for the single-cell system and panel (b) shows them for the four-cell system: The latter has a flat mode between $\Gamma$ and $X^{\prime}$. 


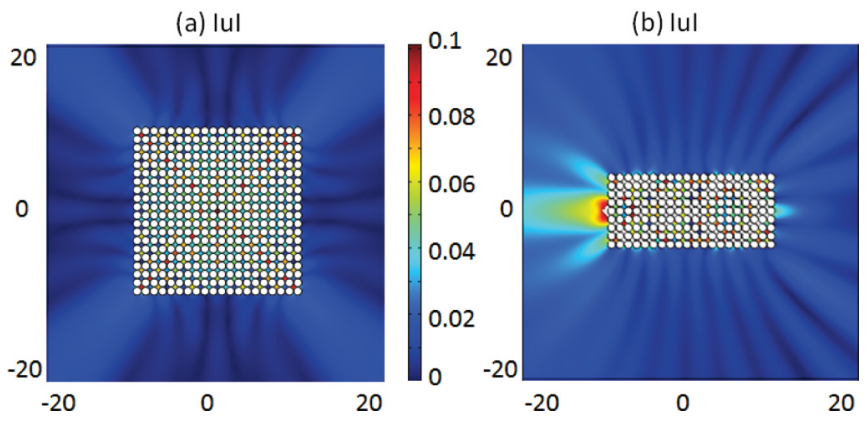

FIG. 9. (Color online) Nearly touching cylinders: (a) $20 \times 20$ circular cylinders, $r=0.475$, pitch 1 , source at $(0,0)$, frequency 1.37; and (b) $9 \times 20$ circular cylinders, $r=0.475$, pitch 1 , source at $(-10.1,0)$, frequency 1.39 .

\section{LARGE CYLINDERS}

To clarify details we illustrate that the analogy between cylinders in the TE polarization and the discrete system can be made sharper. The dispersion relation for large, almost touching cylinders is shown in Fig. 8; the dashed mode along $\Gamma X^{\prime}$ is now almost perfectly flat and the dispersion curves of Fig. 8 become (modulo a multiplicative factor) almost those of the mass-spring model in Fig. 3. Physically one can interpret each Neumann cylinder as a mass connected to its neighbors by a thin wall that acts as a spring ${ }^{20}$ for which the flat band will occur at $\Omega=\pi / 2$; further computations confirm this limit.

As the analogy with the mass spring becomes stronger, the effects of the diagonal cross mode become sharper. Figure 9 shows this for forcing with a square array of cylinders with strongly localized waves along the diagonal; the cross is slightly obscured, visually, by the sheer size of the cylinders. The image is enhanced for the endoscope and the zigzag bouncing of the waves within the cylindrical array is emphasized but again visually obscured by the large cylinders.

\section{ROTATED ARRAY}

For the smaller cylinders the endoscope effect is weaker than for the discrete mass system. As we have just seen, the discrete mass system is a perfect paradigm, but the realistic system has two flaws: First, the critical dispersion curve is no longer dispersionless and has some curvature. Second, the continuous system is relatively small versus the discrete system: $100 \times 100$ cylinders versus $1000 \times 1000$ masses. One option to enhance the effect is to rotate the array of cylinders through an angle $\pi / 4$, thereby facilitating the wave propagation $x_{1}$ direction; see Fig. 10(b). It is worth noticing (a) lul

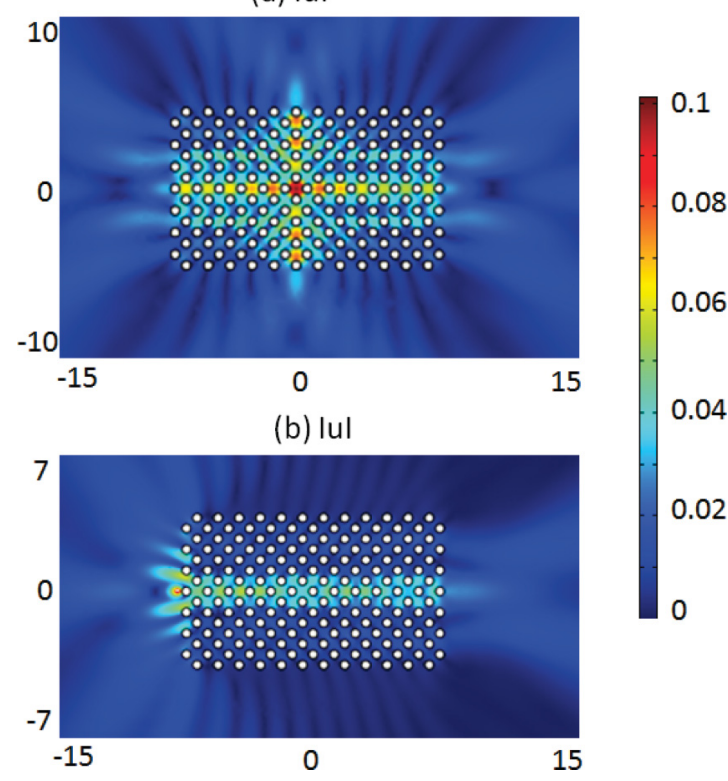

FIG. 10. (Color online) Excitation using a square array rotated through an angle $\pi / 4$ : (a) A cross for an electric line source of normalized frequency $\Omega=2.25$ located at point $(0,0)$ radiating in an isotropic dielectric medium $(\varepsilon=1)$ with a rotated square array (pitch 1) of 200 infinite conducting cylinders (radius $r=0.3$ ) in TE polarization (i.e., Neumann boundary conditions), surrounded by continuous PMLs outside the region $-15<x_{j}<15, j=1,2$. (b) Endoscope effect for the same configuration as in panel (a) but for an electric line source of normalized frequency $\Omega=2.35$ located at point $(-12,0)$.

that with such an orientation of the array, the diagonal cross effect in Fig. 1 is also rotated through an angle $\pi / 4$ [see Fig. 10(a)] to become a vertical cross.

\section{CONCLUDING REMARKS}

We have conclusively shown, using analogies between spring-mass and continuous models, that one must be extra careful when using only the edges of the Brillouin zone instead of its area. While Fermi surfaces ${ }^{21}$ (i.e., dispersion surfaces) contain within them all the information required to completely describe the stop-band structure of a periodic structure, dispersion curves can miss some important features, such as the cross. This standing wave has consequences for the design of practically implementable focusing devices. A possible extension of our study is the analysis of stop bands of absorptive photonic crystals, where the dangers of using the edges of the Brillouin zone are further enhanced. ${ }^{22}$
${ }^{1}$ L. Brillouin, Wave Propagation in Periodic Structures: Electric Filters and Crystal Lattices, 2nd ed. (Dover, New York, 1976).

${ }^{2}$ C. Kittel, Introduction to Solid State Physics, 7th ed. (John Wiley \& Sons, New York, 1996).

${ }^{3}$ J. D. Joannopoulos, S. G. Johnson, J. N. Winn, and R. D. Meade, Photonic Crystals: Molding the Flow of Light, 2nd ed. (Princeton University Press, Princeton, NJ, 2008).
${ }^{4}$ S. D. M. Adams, R. V. Craster, and S. Guenneau, Proc. R. Soc. London, Ser. A 464, 2669 (2008).

${ }^{5}$ J. M. Harrison, P. Kuchment, A. Sobolev, and B. Winn, J. Phys. A 40, 7597 (2007).

${ }^{6}$ P. Markos and C. M. Soukoulis, Wave Propagation: From Electrons to Photonic Crystals and Left-Handed Materials (Princeton University Press, Princeton, NJ, 2008). 
${ }^{7} \mathrm{G}$. W. Milton, The Theory of Composites (Cambridge University Press, Cambridge, 2002).

${ }^{8}$ A. B. Movchan, N. V. Movchan, and C. G. Poulton, Asymptotic Models of Fields in Dilute and Densely Packed Composites (ICP Press, London, 2002).

${ }^{9}$ C. Conca, J. Planchard, and M. Vanninathan, Fluids and Periodic Structures (John Wiley \& Sons, Masson, Paris, 1995).

${ }^{10} \mathrm{M}$. Eastham, The Spectral Theory of Periodic Differential Equations (Scottish Academic Press, Edinburgh, 1973).

${ }^{11}$ S. D. M. Adams, R. V. Craster, and S. Guenneau, Waves Random Complex Media 19, 321 (2009).

${ }^{12}$ A. Figotin and I. Vitebskiy, Waves Random Complex Media 16, 293 (2006).

${ }^{13}$ M. V. Ayzenberg-Stepanenko and L. I. Slepyan, J. Sound Vib. 313, 812 (2008).

${ }^{14}$ R. V. Craster, J. Kaplunov, and J. Postnova, Q. J. Mech. Appl. Math. 63, 497 (2010).
${ }^{15}$ D. J. Colquitt, I. S. Jones, N. V. Movchan, A. B. Movchan, and R. C. McPhedran, Waves Random Complex Media 22, 143 (2012).

${ }^{16} \mathrm{G}$. Floquet, Annales de l'cole Normale Suprieure 12, 47 (1883).

${ }^{17}$ R. V. Craster, J. Kaplunov, E. Nolde, and S. Guenneau, J. Opt. Soc. Am. A 28, 1032 (2011).

${ }^{18}$ S. A. Ramakrishna, Rep. Prog. Phys. 68, 449 (2005).

${ }^{19}$ P. Ferrand, J. Wenger, M. Pianta, H. Rigneault, A. Devilez, B. Stout, N. Bonod, and E. Popov, Opt. Express 16, 6930 (2008).

${ }^{20} \mathrm{M}$. Farhat, S. Guenneau, S. Enoch, G. Tayeb, A. B. Movchan, and N. V. Movchan, Phys. Rev. E 77, 046308 (2008).

${ }^{21}$ N. Ashcroft and N. Mermin, Solid-State Physics (Harcourt College Publishers, New York, 1976).

${ }^{22}$ P. Y. Chen, C. G. Poulton, A. A. Asatryan, M. J. Steel, L. C. Botten, C. Martijn de Sterke, and R. C. McPhedran, New J. Phys. 13, 053007 (2011). 